\title{
Can helmet design reduce the risk of concussion in football?
}

\author{
Technical note
}

\author{
Steven Rowson, Ph.D., ${ }^{1}$ Stefan M. Duma, Ph.D., ${ }^{1}$ Richard M. Greenwald, Ph.D., ${ }^{2,11}$ \\ Jonathan G. Beckwith, M.S., ${ }^{2}$ Jeffrey J. Chu, M.S., ${ }^{2}$ Kevin M. Guskiewicz, Ph.D., ${ }^{3}$ \\ Jason P. Mihalik, Ph.D., Joseph J. Crisco, Ph.D., ${ }^{4}$ Bethany J. Wilcox, B.S., ${ }^{4}$ \\ Thomas W. McAllister, M.D., ${ }^{5}$ Arthur C. Maerlender, Ph.D., ${ }^{6}$ \\ Steven P. Broglio, Ph.D., Brock Schnebel, M.D., ${ }^{7}$ Scott Anderson, B.S., 9 \\ and P. Gunnar Brolinson, D.O. ${ }^{10}$
}

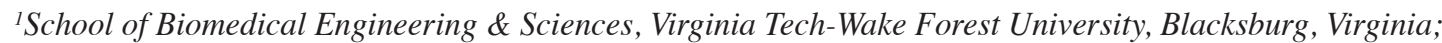
${ }^{2}$ Simbex, Lebanon, New Hampshire; ${ }^{3}$ Matthew Gfeller Sport-Related Traumatic Brain Injury Research Center, University of North Carolina at Chapel Hill, North Carolina; ${ }^{4}$ Department of Orthopaedics, Alpert Medical School of Brown University and Rhode Island Hospital, Providence, Rhode Island; ${ }^{5}$ Department of Psychiatry, Indiana University School of Medicine, Indianapolis, Indiana; ${ }^{6}$ Pediatric Neuropsychological Services, Geisel School of Medicine at Dartmouth, and "Thayer School of Engineering, Dartmouth College, Hanover, New Hampshire; ${ }^{7}$ School of Kinesiology, University of Michigan, Ann Arbor, Michigan; ${ }^{8}$ Departments of Orthopedics and Athletics, ${ }^{9}$ Department of Intercollegiate Athletics, University of Oklahoma, Norman, Oklahoma; and ${ }^{10}$ Edward Via College of Osteopathic Medicine, Blacksburg, Virginia

\begin{abstract}
Of all sports, football accounts for the highest incidence of concussion in the US due to the large number of athletes participating and the nature of the sport. While there is general agreement that concussion incidence can be reduced through rule changes and teaching proper tackling technique, there remains debate as to whether helmet design may also reduce the incidence of concussion. A retrospective analysis was performed of head impact data collected from 1833 collegiate football players who were instrumented with helmet-mounted accelerometer arrays for games and practices. Data were collected between 2005 and 2010 from 8 collegiate football teams: Virginia Tech, University of North Carolina, University of Oklahoma, Dartmouth College, Brown University, University of Minnesota, Indiana University, and University of Illinois. Concussion rates were compared between players wearing Riddell VSR4 and Riddell Revolution helmets while controlling for the head impact exposure of each player. A total of 1,281,444 head impacts were recorded, from which 64 concussions were diagnosed. The relative risk of sustaining a concussion in a Revolution helmet compared with a VSR4 helmet was 46.1\% (95\% CI 28.1\%-75.8\%). When controlling for each player's exposure to head impact, a significant difference was found between concussion rates for players in VSR4 and Revolution helmets $\left(\chi^{2}=4.68, \mathrm{p}=0.0305\right)$. This study illustrates that differences in the ability to reduce concussion risk exist between helmet models in football. Although helmet design may never prevent all concussions from occurring in football, evidence illustrates that it can reduce the incidence of this injury.

(http://thejns.org/doi/abs/10.3171/2014.1.JNS13916)
\end{abstract}

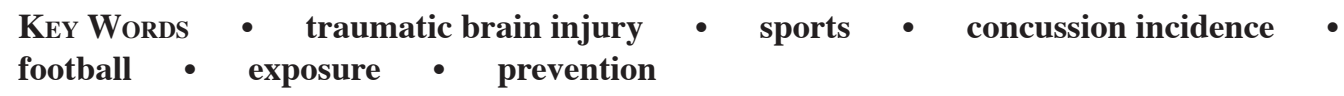

$\mathrm{S}$ PORTS-RELATED concussions were once believed to only result in transient symptoms and neurocognitive impairment. However, recent research has suggested potential links between repetitive concussions and neurodegenerative processes in some athletes. ${ }^{6,12,13}$ This work has led to increased awareness and media attention on the possible long-term effects of sports-related concussions. Of all sports, football accounts for the highest incidence of concussion in the US due to the large number of athletes participating and the nature of the sport. While there is general agreement among experts that concussion incidence can be reduced through rule changes and teaching proper tackling technique, there remains debate as to whether helmet design may also reduce the incidence of concussion. ${ }^{4,11}$ This question is examined retrospectively by analyzing head impact exposure data collected from a population of collegiate football players equipped with 2 different helmet models.

\section{Methods}

Study Population and Data Collection

Between 2005 and 2010, a total of 1833 football players were instrumented with helmet-mounted accelerometer

This article contains some figures that are displayed in color online but in black-and-white in the print edition. 
arrays (HIT System, Simbex). ${ }^{1}$ Data were collected from 8 collegiate football teams: Virginia Tech, University of North Carolina, University of Oklahoma, Dartmouth College, Brown University, University of Minnesota, Indiana University, and University of Illinois. Data collection protocols were approved by each school's institutional review board. Head impact exposure data (severity, frequency, and impact location) were collected for all games and practices that the players participated in, and were paired with clinical data provided by team physicians (diagnosis of concussion). ${ }^{2,3}$ Players were equipped with either a Riddell VSR4 or Riddell Revolution helmet (Fig. 1). Throughout the duration of data collection, a concussion was generally defined as an alteration in mental status resulting from a blow to the head reported by the player or observed by a team's medical staff. All concussions were diagnosed by a certified athletic trainer or team physician at each institution. Given that this is a retrospective analysis of existing data, diagnosis of concussion could not be biased toward a specific helmet type.

\section{Statistical Analysis}

Concussion incidence rates with $95 \%$ CIs were computed for each helmet model by normalizing the number of recorded impacts resulting in diagnosed concussion by the total number of recorded impacts. The relative risk of sustaining a concussion in a Revolution helmet compared with a VSR4 helmet and the associated 95\% CIs were computed. The 99 th percentile acceleration magnitudes resulting from impact were compared between helmet types by player position. A bootstrap technique was used to compute $95 \%$ CIs. In addition, the proportions of concussed players by helmet model were computed with 95\% CIs. The distributions of the number of impacts per player by helmet model were compared using a Wilcoxon

\section{Riddell VSR4}
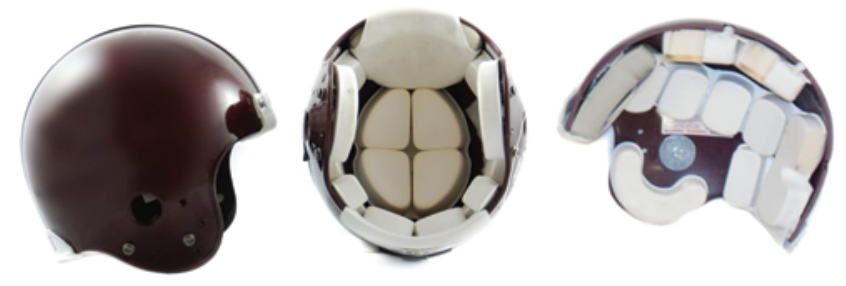

\section{Riddell Revolution}
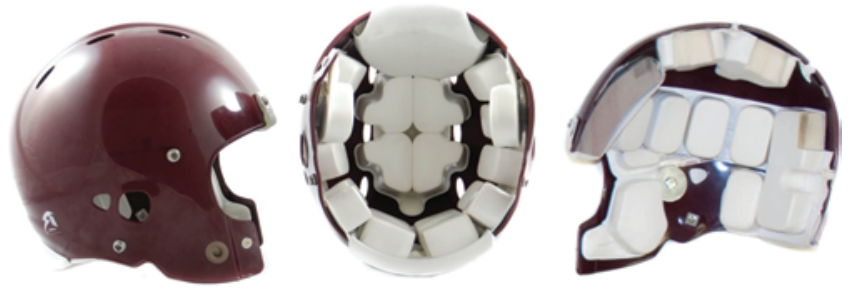

FIG. 1. Exterior, interior, and cross-section of the Riddell VSR4 (upper) and Riddell Revolution (lower) helmets. The Revolution helmet has a greater offset and $40 \%$ thicker foam than the VSR4 helmet. rank-sum test. Furthermore, a Cochran-Mantel-Haenszel analysis was performed to examine the relationship between helmet model and outcome on a per player basis, while controlling for each player's head impact exposure, using an a priori significance threshold of $\mathrm{p}<0.05$.

\section{Results}

A total of 1,281,444 head impacts were recorded, from which 64 concussions were diagnosed. There was a total of 322,725 head impacts to players wearing Riddell VSR4 helmets, including 27 concussions. Players in VSR4 helmets sustained 8.37 concussions $(95 \%$ CI 5.70-12.2) per 100,000 head impacts. There was a total of 958,719 impacts to players wearing Riddell Revolution helmets, including 37 concussions. Players in Revolution helmets sustained 3.86 concussions (95\% CI 2.78-5.34) per 100,000 head impacts. The relative risk of sustaining a concussion in a Revolution helmet compared with a VSR4 helmet was $46.1 \%$ (95\% CI 28.1\%-75.8\%). Overall, players in VSR4 helmets experienced high-magnitude impacts more frequently than players in Revolution helmets (Fig. 2 ). For each player position, the 99th percentile impact was greater for the VSR4 helmet than the Revolution helmet (Table 1).

Among all players, 3.34\% (95\% CI 2.60\%-4.26\%) sustained concussions. A lower percentage of players in Revolution helmets sustained concussions than players in VSR4 helmets, despite players in Revolution helmets experiencing significantly more impacts per season than players in VSR4 helmets $(\mathrm{Z}=-4.95, \mathrm{p}<0.0001)$. Of all players in VSR4 helmets, $4.47 \%$ (95\% CI 3.04\%-6.49\%) sustained concussions. Of all players in Revolution helmets, $2.82 \%$ (95\% CI 2.03\%-3.89\%) sustained concussions. When controlling for each player's exposure to head impact, a significant difference was found between concussion rates for players in VSR4 and Revolution helmets $\left(\chi^{2}=4.68, p=0.0305\right)$.

\section{Discussion}

This retrospective analysis addresses the question of

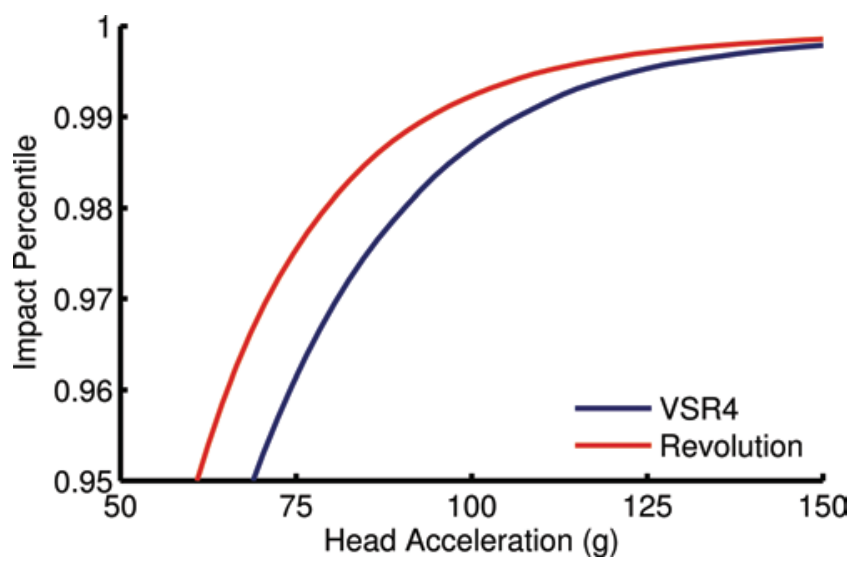

Fig. 2. Comparison of the top 5th percentile of head acceleration magnitudes experienced by players in Riddell VSR4 and Revolution helmets. Players in VSR4 helmets experienced high-magnitude impacts more frequently than players in Revolution helmets. 
TABLE 1: Comparison of 99th percentile head accelerations for each helmet type by player position*

\begin{tabular}{lcc}
\hline \multicolumn{1}{c}{ Position $\dagger$} & VSR4 Helmets & Revolution Helmets \\
\hline defensive back & $101.6(100.3-103.3)$ & $99.1(97.2-101.5)$ \\
defensive lineman & $97.3(95.2-99.2)$ & $89.3(88.5-89.8)$ \\
linebacker & $99.4(97.5-101.1)$ & $96.2(95.0-97.3)$ \\
offensive lineman & $103.3(101.4-105.2)$ & $90.1(89.5-90.7)$ \\
quarterback & $122.7(119.5-125.3)$ & $112.5(107.5-121.2)$ \\
running back & $110.1(107.8-112.2)$ & $105.2(103.4-107.0)$ \\
wide receiver & $106.0(104.3-108.4)$ & $101.0(98.7-103.5)$ \\
\hline
\end{tabular}

* All data given as 99 th percentile head accelerations (g) and $95 \% \mathrm{Cls}$ (in parentheses).

† For every player position, the VSR4 helmet group had a greater 99th percentile head acceleration value than the Revolution helmet group. This is a result of the Revolution helmet better modulating impact energy to reduce head acceleration relative to the VSR4 helmet.

whether helmet design can reduce the incidence of concussion, and reports a $53.9 \%$ reduction in concussion risk associated with the Revolution helmet compared with the VSR4 helmet. This is the first study to show a significant difference in concussion risk between helmet models while utilizing a large cohort and controlling for the number of head impacts each player experienced. In 2006, Collins et al. observed more than 2000 high school players and reported that the Revolution helmet reduced the risk of concussion by $31 \%$ compared with other helmets. ${ }^{5}$ This study was limited because it did not account for impact exposure, and the age of non-Revolution helmets was unknown. In 2012, Rowson and Duma analyzed 9 years of head impact data collected from 308 players and reported that the Revolution helmet reduced the risk of concussion by $85 \%$ compared with the VSR4 helmet. ${ }^{16}$ That investigation addressed the previous limitations of the study of Collins et al. because helmet age was consistent, as each player had been provided with a new helmet of either model. Furthermore, the same team physician made each concussion diagnosis throughout the 9 years, and the head impact exposure of each player was controlled for.

The data presented in this study corroborate and expand upon previous reports of differences in concussion risk by helmet model using a large sample size, systematic medical care, regulated equipment, and the ability to control for the number of times each helmet was impacted. Each institution had a team physician and athletic trainers to monitor and evaluate players during games and practices. Furthermore, data were collected from institutions that take care of the helmets, and replaced helmets on a regular basis. Most importantly, exposure was quantified in terms of the number of head impacts experienced by instrumented players, which provides more valuable information related to the question of helmet design than the total number of players or athletic exposures.

It should be noted that this study is specific to diagnosed concussion rates and does not account for the suspected widespread under-reporting of concussion. Previous studies have suggested that actual concussion rates are 2-10 times greater than diagnosed concussion rates. ${ }^{9,10}$
The diagnosed concussion rates reported in this study are consistent with those previously reported for Division I collegiate football players. ${ }^{7}$ The under-reporting rate was believed to be consistent among helmet models during the span of data collection. Furthermore, all teams included in this study were Division I collegiate football teams. While the distributions of VSR4 and Revolution helmets varied between teams, all teams played Division I competition and analyses of collected data were normalized by impact frequency. For these reasons, the analyses presented in this study are not believed to be sensitive to potential differences between teams. This experiment was not designed to be an epidemiology study, but rather this existing data set was identified by the authors as the best available data to address the question of whether helmet design can influence concussion incidence.

From a biomechanical standpoint, the difference in concussion risk between helmets is logical. A helmet modulates the energy transfer to the head during impact, which dictates the accelerations that the head will experience. These head accelerations result in transient intracranial pressure gradients and neural tissue strain responses, and are correlated with the risk of concussion. ${ }^{8,14}$ Not all helmets are designed equally in their ability to reduce the head accelerations resulting from impact. For matched impacts, the Revolution helmet results in lower head accelerations than the VSR4 helmet (Fig. 3). ${ }^{15,17}$ This reduction of head acceleration in the Revolution helmet reduces the risk of concussion compared with the VSR4 helmet. This point is reinforced by the data shown in Table 1, which demonstrates that players in VSR4 helmets at every evaluated position experienced high-magnitude impacts more frequently than players in Revolution helmets, which represent the impacts that are most likely to result in concussion.

\section{Conclusions}

This exemplar comparison illustrates that differences in the ability to reduce concussion risk exist between helmet models in collegiate football. Helmet designs should be optimized to reduce head acceleration over the continuum of impacts experienced by football players. Helmet design may never prevent all concussions from occurring in football, but the evidence illustrates that it can reduce the incidence of this injury.

\section{Disclosure}

This work was supported in part by grant nos. R01HD048638 and R01NS055020 from the NIH, grant nos. R01CE001254 and 5R49CE000196 from the Centers for Disease Control and Prevention, and grant nos. 07-04 and 14-19 from the National Operating Committee on Standards for Athletic Equipment (NOCSAE). The HIT System technology was developed in part under NIH grant no. R44HD40473. Joseph J. Crisco, Richard M. Greenwald, Jeffrey J. Chu, Jonathan G. Beckwith, and Simbex have a financial interest in the instruments (HIT System, Sideline Response System [Riddell]) that were used to collect the biomechanical data reported in this study. The remaining authors have no financial interests associated with this study.

Author contributions to the study and manuscript preparation include the following. Conception and design: Rowson, Duma, 


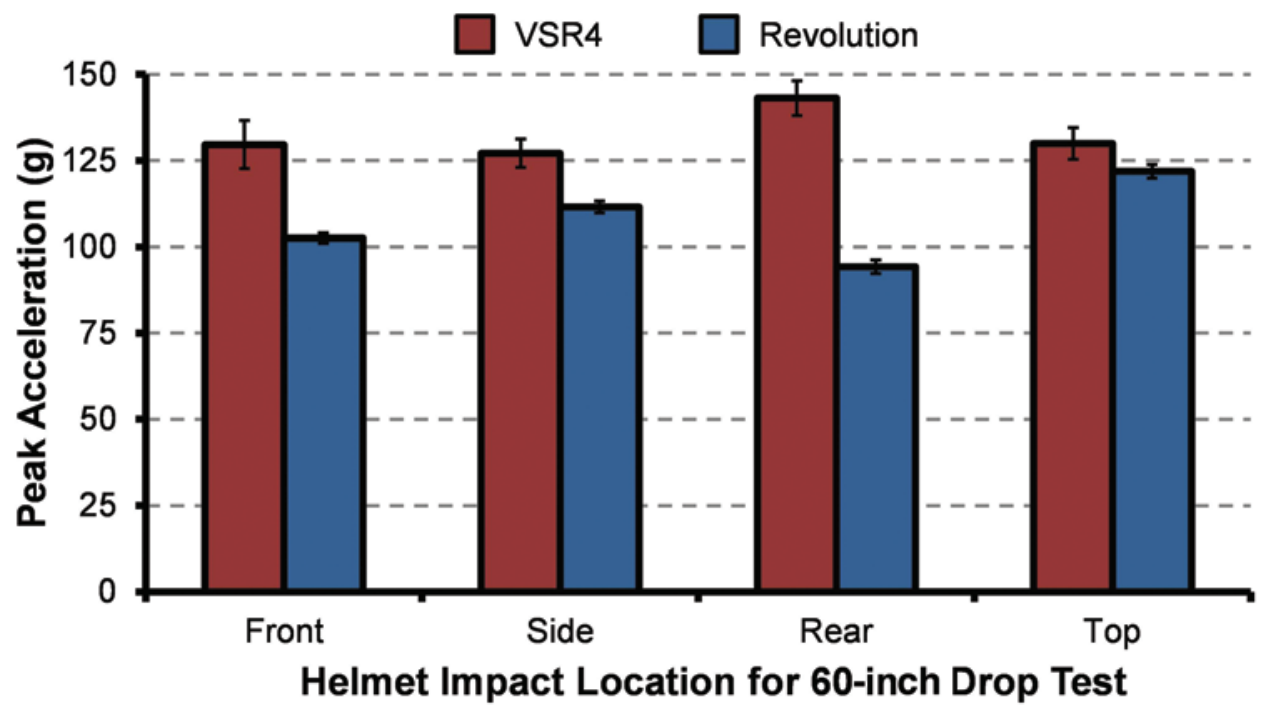

FIG. 3. Comparison of linear acceleration attenuation capability between Riddell VSR4 and Revolution helmets using previously developed laboratory testing methods. ${ }^{11}$ The Revolution helmet better reduced linear head acceleration than the VSR4 helmet for each impact location from a 60-inch drop height. The Revolution helmet has also been shown to better reduce rotational accelerations compared with the VSR4 helmet. ${ }^{12}$ These reductions in head acceleration are associated with a reduction in concussion risk.

Greenwald. Acquisition of data: Rowson, Guskiewicz, Mihalik, Crisco, Wilcox, McAllister, Broglio, Schnebel, Anderson, Brolinson. Analysis and interpretation of data: Rowson, Duma, Greenwald, Beckwith, Maerlender. Drafting the article: Rowson, Duma. Critically revising the article: all authors. Reviewed submitted version of manuscript: all authors. Approved the final version of the manuscript on behalf of all authors: Rowson. Statistical analysis: Rowson. Administrative/technical/material support: Duma, Greenwald, Beckwith, Chu. Study supervision: Duma, Greenwald, Guskiewicz, Mihalik, Crisco, McAllister, Maerlender, Broglio, Schnebel, Anderson, Brolinson.

\section{References}

1. Beckwith JG, Greenwald RM, Chu JJ: Measuring head kinematics in football: correlation between the head impact telemetry system and Hybrid III headform. Ann Biomed Eng 40:237-248, 2012

2. Beckwith JG, Greenwald RM, Chu JJ, Crisco JJ, Rowson S, Duma SM, et al: Head impact exposure sustained by football players on days of diagnosed concussion. Med Sci Sports Exerc 45:737-746, 2013

3. Beckwith JG, Greenwald RM, Chu JJ, Crisco JJ, Rowson S, Duma SM, et al: Timing of concussion diagnosis is related to head impact exposure prior to injury. Med Sci Sports Exerc 45:747-754, 2013

4. Benson BW, McIntosh AS, Maddocks D, Herring SA, Raftery M, Dvorák J: What are the most effective risk-reduction strategies in sport concussion? Br J Sports Med 47:321-326, 2013

5. Collins M, Lovell MR, Iverson GL, Ide T, Maroon J: Examining concussion rates and return to play in high school football players wearing newer helmet technology: a three-year prospective cohort study. Neurosurgery 58:275-286, 2006

6. Gavett BE, Stern RA, McKee AC: Chronic traumatic encephalopathy: a potential late effect of sport-related concussive and subconcussive head trauma. Clin Sports Med 30:179-188, xi, 2011

7. Guskiewicz KM, Weaver NL, Padua DA, Garrett WE Jr: Epidemiology of concussion in collegiate and high school football players. Am J Sports Med 28:643-650, 2000
8. Hardy WN, Mason MJ, Foster CD, Shah CS, Kopacz JM, Yang KH, et al: A study of the response of the human cadaver head to impact. Stapp Car Crash J 51:17-80, 2007

9. Langburt W, Cohen B, Akhthar N, O'Neill K, Lee JC: Incidence of concussion in high school football players of Ohio and Pennsylvania. J Child Neurol 16:83-85, 2001

10. McCrea M, Hammeke T, Olsen G, Leo P, Guskiewicz K: Unreported concussion in high school football players: implications for prevention. Clin J Sport Med 14:13-17, 2004

11. McCrory P, Meeuwisse W, Aubry M, Cantu B, Dvorak J, Echemendia RJ, et al: Consensus statement on concussion in sportthe 4th International Conference on Concussion in Sport held in Zurich, November 2012. Clin J Sport Med 23:89-117, 2013

12. Omalu BI, DeKosky ST, Hamilton RL, Minster RL, Kamboh MI, Shakir AM, et al: Chronic traumatic encephalopathy in a national football league player: part II. Neurosurgery 59: 1086-1093, 2006

13. Omalu BI, DeKosky ST, Minster RL, Kamboh MI, Hamilton $\mathrm{RL}$, Wecht CH: Chronic traumatic encephalopathy in a National Football League player. Neurosurgery 57:128-134, 2005

14. Rowson S, Duma SM: Brain injury prediction: assessing the combined probability of concussion using linear and rotational head acceleration. Ann Biomed Eng 41:873-882, 2013

15. Rowson S, Duma SM: Development of the STAR evaluation system for football helmets: integrating player head impact exposure and risk of concussion. Ann Biomed Eng 39:21302140,2011

16. Rowson S, Duma SM: The Virginia Tech response. Ann Biomed Eng 40:2512-2518, 2012 (Letter)

17. Viano DC, Withnall C, Halstead D: Impact performance of modern football helmets. Ann Biomed Eng 40:160-174, 2012

Manuscript submitted May 3, 2013.

Accepted January 7, 2014

Please include this information when citing this paper: published online January 31, 2014; DOI: 10.3171/2014.1.JNS13916.

Address correspondence to: Steven Rowson, Ph.D., 440 Kelly Hall, 325 Stanger St., Blacksburg, VA 24061. email: srowson@ vt. edu. 